\title{
Front Matter: Volume 9884
}

, "Front Matter: Volume 9884," Proc. SPIE 9884, Nanophotonics VI, 988401 (10 June 2016); doi: 10.1117/12.2242897

SPIE. Event: SPIE Photonics Europe, 2016, Brussels, Belgium 


\title{
PROCEEDINGS OF SPIE
}

\section{Nanophotonics VI}

\author{
David L. Andrews \\ Jean-Michel Nunzi \\ Andreas Ostendorf \\ Editors
}

3-7 April 2016
Brussels, Belgium

Sponsored by

SPIE

Cosponsored by

B-PHOT-Brussels Photonics Team (Belgium)

Research Foundation Flanders (Belgium)

Visit Brussels (Belgium)

Cooperating Organisations

Photonics 21 (Germany)

EOS-European Optical Society (Germany)

KTN-the Knowledge Transfer Network (United Kingdom)

Graphene Flagship (Belgium)

Photonics Public Private Partnership (Belgium)

Published by

SPIE 
The papers in this volume were part of the technical conference cited on the cover and title page. Papers were selected and subject to review by the editors and conference program committee. Some conference presentations may not be available for publication. Additional papers and presentation recordings may be available online in the SPIE Digital Library at SPIEDigitallibrary.org.

The papers reflect the work and thoughts of the authors and are published herein as submitted. The publisher is not responsible for the validity of the information or for any outcomes resulting from reliance thereon.

Please use the following format to cite material from this book:

Author(s), "Title of Paper," in Nanophotonics VI, edited by David L. Andrews, Jean-Michel Nunzi, Andreas Ostendorf, Proceedings of SPIE Vol. 9884 (SPIE, Bellingham, WA, 2016) Six-digit Article CID Number.

ISSN: 0277-786X

ISSN: 1996-756X (electronic)

ISBN: 9781510601291

Published by

SPIE

P.O. Box 10, Bellingham, Washington 98227-0010 USA

Telephone +1 3606763290 (Pacific Time) · Fax +1 3606471445

SPIE.org

Copyright (C) 2016, Society of Photo-Optical Instrumentation Engineers.

Copying of material in this book for internal or personal use, or for the internal or personal use of specific clients, beyond the fair use provisions granted by the U.S. Copyright Law is authorized by SPIE subject to payment of copying fees. The Transactional Reporting Service base fee for this volume is $\$ 18.00$ per article (or portion thereof), which should be paid directly to the Copyright Clearance Center (CCC), 222 Rosewood Drive, Danvers, MA 01923. Payment may also be made electronically through CCC Online at copyright.com. Other copying for republication, resale, advertising or promotion, or any form of systematic or multiple reproduction of any material in this book is prohibited except with permission in writing from the publisher. The CCC fee code is 0277-786X/16/\$18.00.

Printed in the United States of America.

Publication of record for individual papers is online in the SPIE Digital Library.

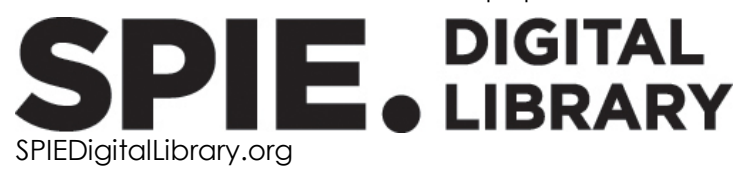

Paper Numbering: Proceedings of SPIE follow an e-First publication model, with papers published first online and then in print. Papers are published as they are submitted and meet publication criteria. A unique citation identifier (CID) number is assigned to each article at the time of the first publication. Utilization of CIDs allows articles to be fully citable as soon as they are published online, and connects the same identifier to all online, print, and electronic versions of the publication. SPIE uses a six-digit CID article numbering system in which:

- The first four digits correspond to the SPIE volume number.

- The last two digits indicate publication order within the volume using a Base 36 numbering system employing both numerals and letters. These two-number sets start with 00, 01, 02, 03, 04, $05,06,07,08,09,0 A, 0 B \ldots$ OZ, followed by 10-1Z, 20-2Z, etc.

The CID Number appears on each page of the manuscript. The complete citation is used on the first page, and an abbreviated version on subsequent pages. 


\title{
Contents
}

\author{
ix Authors \\ xiii Conference Committee
}

LIGHT HARVESTING AND FREQUENCY CONVERSION

988408 Subwavelength resonant antennas enhancing electromagnetic energy harvesting [9884-9]

988409 Mode matching in high non linear susceptibility metamaterials [9884-10]

\section{CAVITIES AND WAVEGUIDES}

$9884 \mathrm{OB} \quad$ How mesoscale lasers can answer fundamental questions related to nanolasers (Invited Paper) [9884-12]

9884 OC Nanoscale photonics using coupled hybrid plasmonic architectures (Invited Paper) [9884-13]

\section{QUANTUM AND NONLINEAR OPTICS IN NANOSTRUCTURES I}

9884 Ol Band diagram of strained graphene nanoribbons [9884-19]

9884 0J Improved nonlinear plasmonic slot waveguide: a full study [9884-20]

QUANTUM AND NONLINEAR OPTICS IN NANOSTRUCTURES II

$9884 \mathrm{OL}$ Architecture, development and implementation of a SWIR to visible integrated upconversion imaging device (Invited Paper) [9884-23]

9884 ON On the emergence of Raman signals characterizing multicenter nanoscale interactions [9884-25]

\section{CONTROL OF NANOSCALE OPTICAL AND ELECTRONIC PROCESSES}

988400 Ultrafast excitonic and charge transfer dynamics in nanostructured organic polymer materials (Keynote Paper) [9884-26]

9884 OP Enhancing Förster nonradiative energy transfer via plasmon interaction (Invited Paper) [9884-27] 
988410 Radiation direction control by optical slot antenna integrated with plasmonic waveguide [9884-37]

988412 Plasmonic hierarchical nanostructures with cascaded field enhancement and their SERS applications [9884-39]

\section{NANOMICROSCOPY}

988413 Monitoring excimer formation of perylene dye molecules within PMMA-based nanofiber via FLIM method [9884-41]

\section{PLASMONICS AND SURFACE NANOSTRUCTURES}

9884 IE Plasmonics in the UV range with Rhodium nanocubes [9884-52]

\section{STRUCTURED LIGHT}

$988411 \quad$ A subwavelength Stokes polarimeter on a silicon chip [9884-57]

$98841 \mathrm{~J}$ Laser controlled deposition of metal microstructures via nondiffracting Bessel beam illumination [9884-58]

FUNCTIONALIZED SENSING

9884 IT Long-term functionalization of optical resonance sensor spots [9884-66]

$98841 \mathrm{U}$ Drastic difference in luminescence stability between amine- and thiol-capped quantum dots treated with $\mathrm{CO}_{2}$ [9884-67]

POSTER SESSION

$9884 \mathrm{IW}$ Photo-induced brightening and broadening effects of gold quantum clusters [9884-70]

9884 IY Using a plasmonic lens to control the emission of electrically excited light [9884-72]

988420 Nonlinear effects in propagation of long-range surface plasmon polaritons in gold strip waveguides [9884-74]

988421 Exciton and multi-exciton dynamics in $\mathrm{CdSe} / \mathrm{Cd}_{1-x} \mathrm{Zn}_{\mathbf{x}} \mathrm{S}$ quantum dots [9884-75]

988422 Fluorescence quantum yield measurement in nanoparticle-fluorophore systems by thermal lens spectroscopy [9884-76] 
988423 CdTe quantum dots: aqueous phase synthesis, stability studies and protein conjugation for development of biosensors [9884-77]

988425 Laser-induced synthesis of a nanostructured polymer-like metal-carbon complexes [9884-79]

988426 The effect of the size of Au nanorods on random laser action in a disordered media of ethylene glycol doped with Rh6G dye [9884-80]

988427 Optical diagnostics of surfaces of single evaporating liquid microdroplet of solutions and suspensions (Best Student Paper Award) [9884-81]

988428 Development of cadmium-free quantum dot for intracellular labelling through electroporation or lipid-calcium-phosphate [9884-82]

9884 2A Grating based hybrid plasmonic waveguide for subwavelength optical confinement with low loss [9884-84]

$98842 \mathrm{~B} \quad$ Enhanced second-harmonic generation driven from magnetic dipole resonance in AlGaAs nanoantennas [9884-85]

9884 2C Deterministic embedding of a single gold nanoparticle into polymeric microstructures by direct laser writing technique [9884-86]

9884 2D Proposed new approach to design all optical AND gate using plasmonic based MachZehnder interferometer for high speed communication [9884-87]

9884 2F Sensing (un)binding events via surface plasmons: effects of resonator geometry [9884-89]

$98842 \mathrm{H} \quad$ Study on structural and optical properties of $\mathrm{TiO}_{2}$ ALD coated silicon nanopillars [9884-91]

988421 Large area gold coated nano-needles fabricated by proximity mask aligner lithography for plasmonic AR-structures [9884-92]

$98842 \mathrm{~L}$ Infrared reduction, an efficient method to control the non-linear optical property of graphene oxide in femtosecond regime [9884-95]

$98842 \mathrm{M}$ Effect of the particle shape on the optical properties of black carbon aggregates [9884-96]

988420 Interpretation of the effect of dielectric spacer on the $\mathrm{ZnO} / \mathrm{Ag}$ structure luminescence intensity [9884-98]

$98842 \mathrm{P}$ Autocorrelation and relaxation time measurements on metal oxide core: dielectric shell beads in an optical trap [9884-99]

$98842 R \quad$ Efficient carrier transfer from graphene quantum dots to GaN epilayers [9884-101]

988425 Sensing characteristics of plasmonic structure based on transferring process of polystyrene nano-beads [9884-102]

$98842 Y \quad$ Fabrication and characterization of the noble metal nanostructures on the GaAs surface [9884-109] 
$98842 Z$ Quantitative comparison of measurement methods for the evaluation of micro- and nanostructures written with 2PP [9884-110]

988431 Aggregation of quantum dots in hybrid structures based on $\mathrm{TiO}_{2}$ nanoparticles [9884-1 12]

988433 Circular dichroism spectroscopy of complexes of semiconductor quantum dots with chlorin e6 [9884-114]

988434 Quantum theory for the nanoscale propagation of light through stacked thin film layers [9884-115]

988435 New design of InGaAs guided-mode resonance photodiode for SWIR low dark current imaging [9884-116]

988437 Plasmonic coupling between metallized fiber tips with sub-wavelength open apertures [9884-118]

988438 Recent progress in plasmonic colour filters for image sensor and multispectral applications [9884-119]

988439 Using femtosecond lasers to modify sizes of gold nanoparticles [9884-120]

9884 3A Influence of the QD luminescence quantum yield on photocurrent in QD/graphene hybrid structures [9884-121]

$98843 \mathrm{C}$ Enhanced fluorescence and aggregation of rhodamine molecules dispersed in a thin polymer film in the presence of plasmonic nanostructures [9884-123]

9884 3D Surface plasmons excited by the photoluminescence of organic nanofibers in hybrid plasmonic systems [9884-124]

9884 3E Dynamic photophoresis-based optical trapping using a spatial light modulator [9884-125]

$98843 \mathrm{~F}$ Luminescence kinetics of the radiative transitions in quantum dots $\mathrm{CdSe} / \mathrm{ZnS}$ in the near field of plasmonic nanoparticles [9884-126]

$98843 G$ FRET efficiency in surface complexes of CdSe/ZnS quantum dots with azo-dyes [9884-127]

$98843 \mathrm{H} \quad$ Modulation of extraordinary optical transmission through nanohole arrays using ultrashort laser pulses [9884-128]

$988431 \quad$ Induced modulation instability of surface plasmon polaritons in a layer structure of subwavelength thickness [9884-129]

$98843 \mathrm{~J} \quad$ Formation of quasiperiodic bimetal thin films with controlled optical and electrical properties [9884-130]

9884 3K Two-level quantum dot susceptibility and polarization in the presence of Coulomb correlations [9884-131]

9884 3L Controllable photo-brightening/photo-darkening of semiconductor quantum dots under laser irradiation [9884-132] 
$98843 \mathrm{P}$ Self-organization and photo-induced formation of cyanine dye aggregates on the plasmonic Ag nanoparticles [9884-136]

9884 3R Plasma model of superconducting crystals [9884-138] 
Proc. of SPIE Vol. $9884988401-8$

Downloaded From: https://www.spiedigitallibrary.org/conference-proceedings-of-spie on 26 Apr 2023 Terms of Use: https://www.spiedigitallibrary.org/terms-of-use 


\section{Authors}

Numbers in the index correspond to the last two digits of the six-digit citation identifier (CID) article numbering system used in Proceedings of SPIE. The first four digits reflect the volume number. Base 36 numbering is employed for the last two digits and indicates the order of articles within the volume. Numbers start with 00, 01, 02, 03, 04, 05, 06, 07, 08, 09, OA, OB...0Z, followed by 10-1Z, 20-2Z, etc.

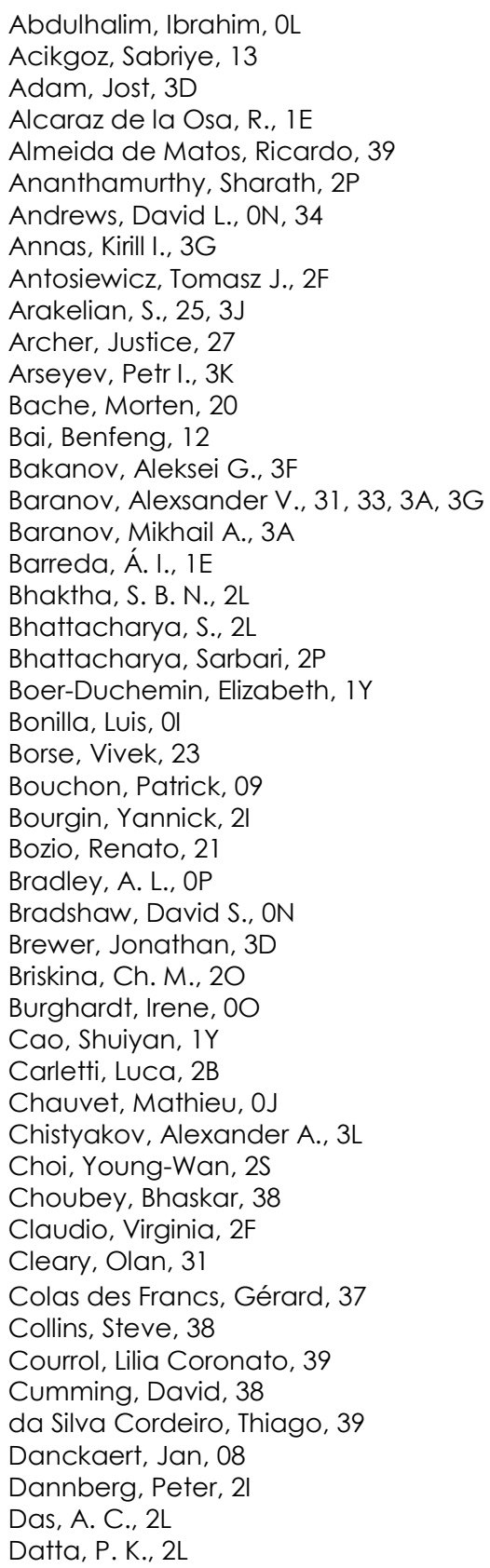

De Angelis, Costantino, 2B

Decombe, Jean-Baptiste, 37

Dehde, Robin, 3H

Demir, Mustafa Muamer, 13

Derkachov, Gennadij, 27

Drampyan, Rafael, $1 \mathrm{~J}$

Drezet, Aurélien, 1Y

Dujardin, Gérald, $1 Y$

Dupuis, Christophe, 35

Eisenbrandt, Pierre, 00

Elsawy, Mahmoud M. R., OJ

Espinosa Soria, A., 11

Everitt, H. O., 1E

Fedorov, Anatoly V., 31, 33, 3A, 3G

Feng, Guoying, 26

Ferreira, M., 22

Fick, Jochen, 37

Finkelstein, G., 1E

Fiutowski, Jacek, 3D

Forbes, Kayn A., 34

Fotiadi, Andrei, 31

Gaponik, N., OP

Gili, Valerio, 2B

Ginis, Vincent, 08

Gladskikh, Igor A., $2 Y$

Gladskikh, Polina V., $2 Y$

Golan, Yuval, OL

González, F., 1E

Grant, James, 38

Griol, Amadeu, 11

Gromova, Yuliya A., 3A, 3 G

Gun'ko, Yurii K., OP, 31

Gutiérrez, Y., $1 \mathrm{E}$

Haacke, Stefan, 00

Haïdar, Riad, 09, 35

Harnisch, Emely Marie, $2 Z$

Hechster, Elad, OL

Helmy, Amr S., OC

Héron, Sébastien, 09

Higgins, L. J., OP

Hou, Tzh-Yin, 28

Huang, Hsiu-Ying, IW

Huang, Hsiu-Ying, 28

Huant, Serge, $1 Y$

Hung, Wei-Ling, 28

Hwang, Jeongwoo, $2 S$

latsunskyi, Igor, $2 \mathrm{H}$

Inci, Mehmet Naci, 13

Istratov, A., 3J 
Itina, T., 3J

lyengar, Shruthi Subhash, 2P

Jaeck, Julien, 35

Jakubczyk, Daniel, 27

Jaworowicz, Katarzyna, 35

Jeon, Seungwon, $2 S$

Jeong, Dae-Cheol, 25

Jozefowski, Leszek, 3D

Jurga, Stefan, $2 \mathrm{H}$

Käll, Mikael, 2F

Kamalieva, Aisylu N., 3C

Karanikolas, V. K., OP

Käsebier, Thomas, 2 I

$\mathrm{Ki}$, Hyun Chul, $2 \mathrm{~S}$

Kim, Doo Gun, $2 S$

Kim, Hong-Seung, $2 S$

Kim, Jineun, 10

Kim, Seon Hoon, $2 S$

Kim, Tae Un, 2S

Kleineberg, Ulf, 3H

Kolesova, Ekaterina P., 31

Kolwas, Krystyna, 27

Kolwas, Maciej, 27

König, Niels, $2 Z$

Korobko, Dmitry, 3l

Krivenkov, Victor, 3L

Kucherik, A., 25, 3J

Kumar, Mukesh, 2A

Kumar, Santosh, 2D

Kundelev, Evgeny V., 33

Kutrovskaya, S., 25, 3J

Lai, Ngoc Diep, 2C

Lavrinenko, Andrei, 20

Le Moal, Eric, $1 Y$

Ledoux-Rak, Isabelle, 2C

Lee, Chang-Won, 10

Leißner, Till, 3D

Leo, Giuseppe, 2B

Leonov, Nikita, $1 \mathrm{~J}$

Lequeux, Médéric, $1 Y$

Lesnyak, V., OP

Li, P., $1 \mathrm{E}$

Lin, Charles, OC

Lin, Cheng-An J., 1W, 28

Lin, Chia-Hui, IW

Lin, Tzu-Neng, 2R

Lippi, G. L., OB

Liu, J., $1 \mathrm{E}$

Liu, Ying-Feng, 28

Locatelli, Andrea, 2B

Lotin, A. A., 20

Lysenko, Oleg, 20

Maiti, R., 2L

Makov, Guy, OL

Malureanu, Radu, 20

Maman, Nitzan, $\mathrm{OL}$

Manshina, A., 25

Mantsevich, Vladimir N., 3K

Markushev, V. M., 20

Marocico, C. A., OP
Martínez, Alejandro, 11

Maslov, Vladimir G., 31, 33, 3A, 3G

Maslova, Natalya S., 3K

Melnik, Roderick, 0 I

Merden Santiago, Svette Reina, 2R

Méry, Stéphane, 00

Michaelis, Dirk, 21

Minotto, Alessandro, 21

Moiseev, Sergey, 31

Mondal, S., 2L

Montes, Kevin, 2C

Moreno, F., $1 \mathrm{E}$

Moshkalev, Stanislav A., 3A

Murphy, G. P., OP

Myndrul, Valerii, 2H

Nabiev, Igor R., 1U, 3L

Nabiullina, Rezida D., 3P

Nazabal, Virginie, OJ

Netesova, Nadezhda P., 3R

Ngo, Hoang Minh, 2C

Nguyen, Dam Thuy Trang, 2C

Nissim, Nimrod, OL

Orlova, Anna O., 31, 33, 3A, 3G

Ortiz, D., 1E

Osipov, A., 25, 3J

Ostendorf, Andreas, IT

Oumbe Tekam, Gabin, 08

Parbrook, P. J., OP

Pardo, Fabrice, 35

Park, Yeonsang, 10

Parthasarathi, Praveen, 2P

Pavlenko, Mykola, 2H

Pearce, Kellie, 3H

Pelissier, Aurélien, 2C

Pelouard, Jean-Luc, 35

Pinton, Nadia, 38

Piscitelli, V., 22

Polkehn, Matthias, 00

Porfirev, Aleksey P., 3E

Portier, Benjamin, 35

Povolotckaia, A., 25

Povolotskiy, A., 25

Prabhakar, Sanjay, 01

Puccioni, G. P., OB

Ray, S. K., 2L

Renversez, Gilles, 0J

Reznik, Ivan A., 3A

Righetto, Marcello, 21

Rocco, Davide, 2B

Rodríguez-Fortuño, Francisco J., 1 I

Rogach, A. L., OP

Roh, Young-Geun, 10

Rubahn, Horst-Günter, 3D

Rumyantsev, S. I., 20

Ryzhkov, M. V., 20

Sadawana, Mayur, 23

Saetchnikov, Anton V., $1 T$

Saetchnikov, Vladimir A., IT

Saha, S., 2 L

Saiz, J. M., 1 E 
Samad, Ricardo Elgul, 39

Samokhvalov, Pavel S., 1U, 3L

Sanz, J. M., 1E

Sarusi, Gabby, OL

Schmidt, Jürgen, $3 \mathrm{H}$

Schmitt, Robert, $2 Z$

Schweiger, Gustav, $1 T$

Seetharamdoo, Divitha, 08

Selvan, Rekha, 2P

Sharma, Anuj, 2A

Sharma, Tarun, 2A

Shen, Ji-Lin, 2R

Shin, Jae Cheol, 25

Silva, Flávia Rodrigues de Oliveira, 39

Singh, Lokendra, 2D

Skidanov, Roman V., 3E

Skorupski, Krzysztof, 2M

Smyntyna, Valentyn, $2 \mathrm{H}$

Sobolewska, Elżbieta K., 3D

Solodar, Assi, OL

Späth, Christian, 3H

Spreen, Anika, 3H

Srivastava, Rohit, 23

Starovoytov, Anton A., 3P

Su, Yiwen, OC

Susha, A. S., OP

Tal, Amir, OL

Tamura, Hiroyuki, 00

Tarasov, A. P., 20

Tcherniavskaia, Elina A., $1 T$

Templeman, Tzvi, OL

Tong, Quang Cong, 2C

Toropov, Nikita A., 2Y, 3C, 3F, 3P

Tretyachenko, Anna, 3L

Valdivia-Valero, Francisco J., 37

Vartanyan, Tigran A., 1 J, 2Y, 3C, 3F, 3J

Verdun, Michaël, 35

Vieira, Nilson Dias, 39

Visoly-Fisher, Iris, OL

Vitenberg, Vladimir, OL

Vokhmintcev, Kirill V., $1 \mathrm{U}$

Wang, Shutong, 26

Wang, T., OB

Watson, A. M., 1E

Wendl, Maximilian, 3H

Williams, Mathew D., ON, 34

Woźniak, Mariusz, 27

Yin, Jiajia, 26

Yuan, Chi-Tsu, 2R

Zeitner, Uwe D., 2

Zhang, Hong, 26

Zhang, Hua, 26

Zhang, X., OP

Zhang, $X_{\text {., }} 1 \mathrm{E}$

Zhou, Shouhuan, 26

Zhu, Zhendong, 12

Zlatov, Andrei S., 3A

Zolotovskii, Igor, 3

Proc. of SPIE Vol. $9884988401-11$ 
Proc. of SPIE Vol. $9884988401-12$

Downloaded From: https://www.spiedigitallibrary.org/conference-proceedings-of-spie on 26 Apr 2023 Terms of Use: https://www.spiedigitallibrary.org/terms-of-use 


\section{Conference Committee}

Symposium Chairs

Francis Berghmans, Vrije Universiteit Brussel (Belgium)

Jürgen Popp, Leibniz-Institut für Photonische Technologien e.V.

(Germany)

Ronan Burgess, European Commission (Belgium)

Peter Hartmann, SCHOTT AG (Germany)

Honorary Symposium Chair

Hugo Thienpont, Vrije Universiteit Brussel (Belgium)

Conference Chairs

David L. Andrews, University of East Anglia (United Kingdom)

Jean-Michel Nunzi, Queen's University (Canada)

Andreas Ostendorf, Ruhr-Universität Bochum (Germany)

Conference Programme Committee

Angus J. Bain, University College London (United Kingdom)

Mario Berberan-Santos, Universidade de Lisboa (Portugal)

Renato Bozio, Università degli Studi di Padova (Italy)

Céline Fiorini-Debuisschert, Commissariat à l'Énergie Atomique (France)

Vincent Ginis, Vrije Universiteit Brussel (Belgium)

Yuval Golan, Ben-Gurion University of the Negev (Israel)

Erez Hasman, Technion-Israel Institute of Technology (Israel)

Yasushi Inouye, Osaka University (Japan)

Gediminas Juzeliunas, Vilnius University (Lithuania)

Martti Kauranen, Tampere University of Technology (Finland)

Satoshi Kawata, Osaka University (Japan)

Francois Lagugné-Labarthet, The University of Western Ontario

(Canada)

Isabelle Ledoux-Rak, Ecole Normale Supérieure de Cachan (France)

Christoph Lienau, Carl von Ossietzky Universität Oldenburg (Germany)

Nazario Martin, Universidad Complutense de Madrid (Spain)

Raúl J. Martín-Palma, Universitdad Autónoma de Madrid (Spain)

Jesper Mork, Technical University of Denmark (Denmark)

Michel Orrit, Leiden University (Netherlands)

Carsten Reinhardt, Laser Zentrum Hannover e.V. (Germany)

Anatoly V. Zayats, King's College London (United Kingdom) 


\section{Session Chairs}

1 Near-field Optics

Angus J. Bain, University College London (United Kingdom)

2 Light Harvesting and Frequency Conversion

Angus J. Bain, University College London (United Kingdom)

3 Cavities and Waveguides

David L. Andrews, University of East Anglia (United Kingdom)

4 Quantum and Nonlinear Optics in Nanostructures I

Jean-Michel Nunzi, Queen's University (Canada)

5 Quantum and Nonlinear Optics in Nanostructures II

Angus J. Bain, University College London (United Kingdom)

6 Control of Nanoscale Optical and Electronic Processes

Jean-Michel Nunzi, Queen's University (Canada)

$7 \quad$ Nanomanipulation with Light

Christoph Lienau, Carl von Ossietzky Universität Oldenburg (Germany)

Jean-Michel Nunzi, Queen's University (Canada)

8 Surface Plasmons and Devices

Anatoly V. Zayats, King's College London (United Kingdom)

9 Nanomicroscopy

Andreas Ostendorf, Ruhr-Universität Bochum (Germany)

10 Photoactive Arrays

Andreas Ostendorf, Ruhr-Universität Bochum (Germany)

11 Plasmonics and Surface Nanostructures

Mario Berberan-Santos, Universidade de Lisboa (Portugal)

12 Structured Light

David L. Andrews, University of East Anglia (United Kingdom)

13 Nanoscale Optics

Andreas Ostendorf, Ruhr-Universität Bochum (Germany)

14 Functionalized Sensing

Mario Berberan-Santos, Universidade de Lisboa (Portugal) 